\title{
Photon Gas at the Planck Scale within the Doubly Special Relativity
}

\author{
W.S. Chung ${ }^{a, b}$, A.M. Gavrilik ${ }^{c, d}$, A.V. Nazarenko ${ }^{c, e}$ \\ ${ }^{a}$ Department of Physics and Research Institute of Natural Science, \\ College of Natural Science, \\ Gyeongsang National University, Jinju 660-701, Korea \\ ${ }^{b}$ mimip44@naver.com \\ ${ }^{c}$ Bogolyubov Institute for Theoretical Physics of NAS of Ukraine, \\ 14-b, Metrolohichna str., Kyiv 03143, Ukraine \\ domgavr@bitp.kiev.ua \\ ${ }^{e}$ nazarenko@bitp.kiev.ua
}

\begin{abstract}
Within the approach to doubly special relativity (DSR) suggested by Magueijo and Smolin, a new algebraically justified rule of so-called $\kappa$-addition for the energies of identical particles is proposed. This rule permits to introduce the nonlinear $\kappa$ dependent Hamiltonian for one-mode multi-photon (sub)system. On its base, with different modes treated as independent, the thermodynamics of black-body radiation is explored within DSR, and main thermodynamic quantities are obtained. In their derivation, we use both the analytical tools within mean field approximation (MFA) and numerical evaluations based on exact formulas. The entropy of one-mode subsystem turns out to be finite (bounded). Another unusual result is the existence of threshold temperature above which radiation is present. Specific features of the obtained results are explained and illustrated with a number of plots. Comparison with some works of relevance is given.

Keywords: doubly special relativity; Planck energy; black body radiation; $\kappa$ addition rule; deformed bosons; photons; bounded radiation energy and entropy; modified Planck law; threshold temperature
\end{abstract}

PACS: 03.30.+p; 05.30.-d; 05.30.Jp; 05.70.Ce; 14.70.Bh; 42.50.Ar 


\section{Introduction}

Recently, the deformation of the special relativity was accomplished so that it might jointly admit two invariant fundamental scales: one of them being the speed of light, and the other - an energy scale naturally identifiable with Planck energy [1, 2, 3, 4, 15]. This deformed theory is usually called a doubly special relativity (DSR) because it has two invariant scales. The DSR is important when one aims to describe the particles' dynamics at very high energies approaching the Planck scale. It is believed that at this scale the spacetime structure can be influenced by effects of quantum gravity.

Initially DSR was introduced in an algebraic way, using certain quantum deformations of the Lorentz group [3, 4]. Later it was also derived by taking as starting point a few physical postulates, including the requirement that it should reduce to special relativity if the low-energy limit is applied [1, 2].

In the ordinary special relativity, the momentum changes under the Lorentz transformation with the infinitesimal boost generator $J$ as

$$
\delta p_{0}=\left\{J, p_{0}\right\}=p_{1}, \quad \delta p_{1}=\left\{J, p_{1}\right\}=p_{0},
$$

where we consider $1+1$ dimensions, and $\{$,$\} denotes the Poisson bracket. The DSR model$ by Magueijo-Smolin (MS model) [5] takes the form

$$
\delta p_{0}=\left\{J, p_{0}\right\}=\left(1-\frac{p_{0}}{\kappa}\right) p_{1}, \quad \delta p_{1}=\left\{J, p_{1}\right\}=p_{0}-\frac{p_{1}^{2}}{\kappa},
$$

with $\kappa$ the Planck energy. Due to the factor $1-p_{0} / \kappa$, for $p_{0}=\kappa$ we have $\delta p_{0}=0$, and thus the invariance of Planck energy. Under the transformation (2), one has the invariant quantity $p^{2} /\left(1-p_{0} / \kappa\right)^{2}$, where $p^{2}=p_{0}^{2}-p_{1}^{2}$.

The DSR models imply that the momentum of a particle transforms nonlinearly under the Lorentz group. In this respect, there is a variety of ways to realize nonlinear representations of the Lorentz group obeying the above-mentioned postulates, but, to develop the theory there are two basic routes: working in ordinary spacetime, or to explore a spacetime involving non-commuting coordinates. In the noncommutative case, for defining the Hamiltonian classical dynamics, noncanonical Poisson brackets are used. It is clear that, depending on the choice of initial assumptions, the explored models may lead to differing physical consequences or predictions.

In this paper we propose a new, DSR-inspired rule of " $\kappa$-addition" for the energies of particles (Section 2), study its properties, and then introduce the corresponding Hamiltonian for one-mode multi-photon system. On its base, assuming independence of different modes, we explore in Sec. 3 the thermodynamics of black-body radiation in the framework of DSR and derive basic thermodynamic quantities. Our calculations lead to rather unusual results, whose special features are analyzed and illustrated with plots. In the final section, main consequences and conclusions are presented, along with brief comparison with respective aspects in some related works. 


\section{New Addition Rule for Energies and the Hamilto- nian}

The exact dispersion relation for DSR (Magueijo-Smolin model) [6] is

$$
\frac{E^{2}-p^{2}}{(1-E / \kappa)^{2}}=m^{2},
$$

where $p_{0}=E$ and $p=|\vec{p}|$ and $m$ is the rest mass. This relation is transformed into the ordinary dispersion relation through the (direct/inverse) map

$$
\begin{aligned}
\epsilon=\frac{E}{1-E / \kappa}, & \vec{\pi}_{i}=\frac{\vec{p}_{i}}{1-E / \kappa}, \\
E=\frac{\epsilon}{1+\epsilon / \kappa}, & \vec{p}_{i}=\frac{\vec{\pi}_{i}}{1+\epsilon / \kappa},
\end{aligned}
$$

so that we have $\epsilon^{2}-\pi^{2}=m^{2}$.

Eq. (5) shows that $E=\kappa$ in the limit $\epsilon \rightarrow \infty$, and from $\epsilon \geq 0$ in Eq. (5) we have $E \leq \kappa$, which implies that there exists a maximum energy in Nature.

Instead of the energy conservation corresponding to the undeformed energy, $\epsilon_{\text {tot }}=$ $\epsilon_{1}+\epsilon_{2}$, we propose the new conservation of energy in DSR theory as

$$
E_{\text {tot }}=E_{1} \oplus_{\kappa} E_{2}
$$

with the " $\kappa$-addition"

$$
a \oplus_{\kappa} b=\frac{\frac{a}{1-a / \kappa}+\frac{b}{1-b / \kappa}}{1+\frac{1}{\kappa}\left(\frac{a}{1-a / \kappa}+\frac{b}{1-b / \kappa}\right)}
$$

which can also be written as

$$
\frac{1}{\frac{1}{a \oplus_{\kappa} b}-\frac{1}{\kappa}}=\frac{1}{\frac{1}{a}-\frac{1}{\kappa}}+\frac{1}{\frac{1}{b}-\frac{1}{\kappa}} .
$$

It is clear that $\kappa$-addition reduces to ordinary addition in the limit $\kappa \rightarrow \infty$.

Properties of $\kappa$-Addition: 1) The $\kappa$-addition is commutative: $a \oplus_{\kappa} b=b \oplus_{\kappa} a$; 2) it is associative: $\left(a \oplus_{\kappa} b\right) \oplus_{\kappa} c=a \oplus_{\kappa}\left(b \oplus_{\kappa} c\right)$; 3) there exists the 0-identity: $\left.a \oplus_{\kappa} 0=a ; 4\right)$ there exists the inverse element for $a$ denoted by $\ominus_{\kappa} a$ : $a \oplus_{\kappa}\left(\ominus_{\kappa} a\right)=0$, which gives $\ominus_{\kappa} a=-\frac{a}{1-2 a / \kappa}$; 5) the $\kappa$-addition of $\kappa$ and any element $a$ gives $\kappa: a \oplus_{\kappa} \kappa=\lim _{b \rightarrow \kappa} a \oplus_{\kappa} b=\kappa$. The last property implies that Planck energy is the maximum energy: $E \oplus_{\kappa} \kappa=\kappa$. Besides, for any positive energies $E_{1}, E_{2}(\neq \kappa)$ we know that $E_{1} \oplus_{\kappa} E_{2}<\kappa$. Thus, Planck energy is the maximum energy as the light speed is the maximum velocity.

Photons at the Planck Scale. Photon obeys the deformed dispersion relation at the Planck scale, which gives the same one as in the undeformed theory, $E=p$. 
But, the energy of photon obeys the deformed law of the energy conservation given by $\kappa$-addition. Thus, the total energy for $n$ photons differs from the $n$ times the energy of single photon, $E_{n \text { photons }} \neq n E_{1 \text { photon }}$.

For example, the energy for two identical photons at the Planck scale obeys

$$
E_{2 \text { photons }}=E_{1 \text { photon }} \oplus_{k} E_{1 \text { photon }}=\frac{2 E_{1 \text { photon }}}{1+E_{1 \text { photon }} / \kappa} .
$$

If we set

$$
n \odot_{\kappa} a=\underbrace{a \oplus_{\kappa} a \oplus_{\kappa} \cdots \oplus_{\kappa} a}_{n}
$$

the energy of $n$ photons is given as

$$
E_{n \text { photons }}=n \odot_{\kappa} E_{1 \text { photon }}=\frac{n E_{1 \text { photon }}}{1+(n-1) E_{1 \text { photon }} / \kappa}
$$

where we require $E_{1 \text { photon }}=h \nu \leq \kappa$. Quite interestingly, we find

$$
\lim _{n \rightarrow \infty} E_{n \text { photons }}=\kappa \text {. }
$$

Thus, the Hamiltonian for photon at Planck scale is

$$
H=\frac{N h \nu}{1+(N-1) h \nu / \kappa}
$$

where the photon creation/destruction operators obey

$$
\left[a, a^{\dagger}\right]=1, \quad N=a^{\dagger} a
$$

and the photon number state is taken as $N|n\rangle=n|n\rangle, n=0,1,2, \ldots$

The single-mode (or monochromatic) multi-photon Hamiltonian (12) coupled with usual bosonic formulas (13) principally differs from the familiar free or linear one $H=N h \nu$ : being essentially nonlinear (with anharmonicities of all orders) it implies highly nontrivial self-interaction within each one mode. In other words, the imposed special rule of $\kappa$ addition given in (10), naturally induces nontrivial self-interaction seen in the Hamiltonian (12). Clearly, the strength of nonlinearity is controlled by $\kappa$.

Similarity with a Class of Deformed Oscillators. It is worth noting that the nonlinear Hamiltonian (12) can also be obtained from a different standpoint beyond the DSR, namely within the theory of deformed oscillators (deformed bosons). That is, the particular deformed oscillator (DO) whose creation/destruction operators $\tilde{a}, \tilde{a}^{\dagger}$ obey the relations

$$
\left[\tilde{a}, \tilde{a}^{\dagger}\right]=\phi_{\kappa}(N+1)-\phi_{\kappa}(N), \quad \phi_{\kappa}(N) \equiv \tilde{a}^{\dagger} \tilde{a}=\frac{N}{1+\kappa^{-1}(N-1) h \nu}
$$


defined by the respective deformation structure function (DSF) $\phi_{\kappa}(N)$ (for the concept of DSF see e.g. [7]), here with the deformation parameter $h \nu / \kappa$. It leads to the free Hamiltonian of deformed bosons of the form

$$
\tilde{H}=h \nu \tilde{a}^{\dagger} \tilde{a}=h \nu \phi_{\kappa}(N),
$$

which coincides with that in (12). In this case, self-interaction is embodied in deformation. Clearly, if $\kappa^{-1} \rightarrow 0$ both $H$ and $\tilde{H}$ reduce to the linear Hamiltonian of usual photons.

The explicit form of the DSF in (14) shows its rational dependence on the excitation number operator $N$. A very similar DO, whose $\operatorname{DSF} \phi_{\mu}(N)=N /(1+\mu N)$ also has rational type $N$-dependence, is known as the $\mu$-oscillator of Jannussis [8]. What is important, DOs with rational nonlinearity principally differ from the best known one-, two-parameter Fibonacci DOs [9, 10, 11] based on DSFs of exponential type. The latter admit the 3-, 4- and 5-parameter (exponential type) extensions given in [12, 13, 14, and all the three belong to the Fibonacci class of DOs as well [15]. On the contrary, DOs with rational nonlinearity of DSF - the $\mu$-oscillator and alike - are not Fibonacci, but "quasi-Fibonacci" ones [15].

There are a few papers exploring diverse aspects of $\mu$-oscillator and of the related $\mu$ analogs of Bose gas model. Besides the already mentioned [8, 15], let us quote the works developing $\mu$-Bose gas model in the context of $n$-particle distributions and correlation function intercepts [16, 17], or from the viewpoint of $\mu$-deformed thermodynamics [18]. Most recently, an application of (the condensate of) $\mu$-Bose gas has been proposed [19] for effective modeling of main properties of dark matter haloes surrounding dwarf galaxies.

\section{Black-body Radiation at Planck Scale}

In principle, there are different possibilities of describing the modified radiation spectrum, consisting of the infinite number of photon modes, corresponding to various frequencies. Most reasonable point of view, proved in [20] for the DSR theories of many-particle systems, suggests to preserve the standard additive form of a total energy and, therefore, to neglect non-local interactions among different modes. In practice, we use the $\kappa$-addition for the identical particles only, i.e. the photons of the same wave length. Thus, the $\kappa$-addition, mathematically formulated for the arbitrary distanced particles, induces a supplemental (non-local) attraction among monochromatic photons, which reduces their total energymass. At the same time, the photon density turns out here to be of order of Planckian density, $\kappa^{3}(\hbar c)^{-3}$.

Under these conditions, we do not consider the soccer-ball problem of DSR [21], concerned mainly with the macroscopic bodies, and discard it due to focusing on the dense (and compact) system, consisting of the non-interacting subsets of identical light quanta. However, similarly to the other DSR theories, the terms of $1 / \kappa$-expansion of a total energymass would diverge by the power law with the growing number of photons [21, while an exact expression does not exceed $\kappa$. This is related with a peculiarity of accounting for supplemental attraction, generated by the modified energy-momentum addition. 
Applying the Planck-scale restriction only to the subsystem of identical photons with the same cyclic frequency $\omega$ and the photon (occupation) number $n_{\omega}$, we write

$$
E\left(\left\{n_{\omega}\right\}\right)=\sum_{\omega} E_{\omega, n}, \quad E_{\omega, n}=\frac{\hbar \omega n_{\omega}}{1+\hbar \omega\left(n_{\omega}-1\right) / \kappa},
$$

where summation operation reflects discreteness of the spectrum in a finite volume $V$; dispersion relation in three space dimensions is also assumed to be $\omega=c|\mathbf{k}|$, where $\mathbf{k}$ is the wave vector.

We see that $E_{\omega, n}<\hbar \omega n_{\omega}$ in general. It physically means a presence of an additional (long-range) attraction among identical photons, which is growing together with $n_{\omega}$. Thus, a photon emission requires a higher temperature regime, comparing the temperature with a characteristic energy of the order of $\kappa$ in magnitude, to overcome the attraction barrier.

On the other hand, the individual energy of photon $\hbar \omega$ should be restricted by condition $\hbar \omega_{\max }=\kappa$, because $E_{\omega, n}=\kappa n_{\omega} /\left(n_{\omega}-1+\kappa / \hbar \omega\right)>\kappa$ at $\hbar \omega \gg \kappa$, that is beyond DSR ideology.

We admit that these restrictions can also appear within other systems of bosons with supplementary attraction, even disconnected with the (modified) special relativity theory. Further on, we are going to describe the thermodynamic properties of such systems.

Grand Partition Function. Formula (15) is used to determine the partition function $\mathcal{Z}^{\text {tot }}$ of the photon gas in volume $V$ within grand canonical ensemble, with temperature $T=\beta^{-1}$, fugacity $z$, and statistical weights defined by the Gibbs measure $\exp \left(-\beta E_{\omega, n}\right)$. Since the photon modes determined by various $\omega$ are viewed as distinct, the function $\mathcal{Z}^{\text {tot }}$ is given as

$$
\begin{gathered}
\ln \mathcal{Z}^{\text {tot }}=\sum_{\omega} \ln \mathcal{Z}_{\omega}, \\
\mathcal{Z}_{\omega}=\mathrm{e}^{-\beta \kappa} \sum_{n=0}^{\infty} z^{n} \exp \left(\beta \kappa \frac{1-\mu_{\omega}}{1+\mu_{\omega}(n-1)}\right), \quad \mu_{\omega}=\frac{\hbar \omega}{\kappa} .
\end{gathered}
$$

Note that the (mode or) $\omega$-dependence of the deformation parameter $\mu_{\omega}$ is quite similar to the momentum dependence of deformation parameter in [22].

Since the values of $E_{\omega, n}$ at $n_{\omega} \geq 1$ lie within the energy band of finite width, $\hbar \omega \leq E_{\omega, n} \leq$ $\kappa$, the series (17) diverges at $z=1$. Thus, the presence of fugacity $z<1$ guarantees the partition function convergence. Also, $z$ can be determined by negative chemical potential $\nu=-|\nu|$ so that $z=\exp (\beta \nu)$, what corresponds to a system with effective attraction.

Main peculiarity of calculating (17) is related with a factor $\sim 1 /\left(1+\mu_{\omega} n\right)$ in the Gibbs measure. Its role can be statistically evaluated by introducing an order parameter (mean field):

$$
\sigma\left(z, x, \mu_{\omega}\right)=\frac{\mathrm{e}^{-x}}{\mathcal{Z}_{\omega}} \sum_{n=0}^{\infty} \frac{1-\mu_{\omega}}{1+\mu_{\omega}(n-1)} z^{n} \exp \left(x \frac{1-\mu_{\omega}}{1+\mu_{\omega}(n-1)}\right) .
$$

Hereafter $x \equiv \beta \kappa$, and $\mathcal{Z}_{\omega}=\mathcal{Z}\left(z, x, \mu_{\omega}\right)$ is also assumed. 


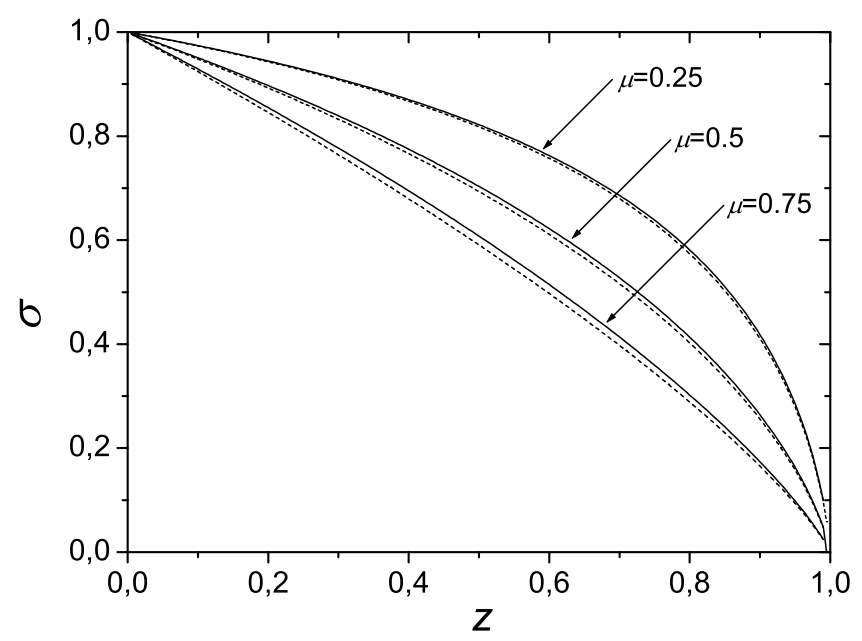

Figure 1: Mean field $\sigma$ versus fugacity $z$ at different values of $\mu$. Solid curves represent the exact dependencies (18), computed numerically at $\beta \kappa=0.1$. Dashed curves, closest to the corresponding solid ones, are obtained analytically in MFA (21) i.e., at $\beta \kappa=0$.

One can verify numerically that $\sigma \approx 1$ at $x \gg 1$. Non-trivial thermodynamics appears at $x \ll 1$ that requires the temperature $T$ higher than $\kappa$. If $0<\mu<1$, then $0 \leq \sigma \leq 1$. Setting $\mu>1$, there is a region of $(z, x)$ for which $\sigma<0$.

We perform the following calculations assuming that $x<1$ and $0<\mu<1$. The order parameter existence permits us to evaluate the partition function and thermodynamic functions in the mean field approximation (MFA), replacing the factor $\left(1-\mu_{\omega}\right) /\left(1+\mu_{\omega}(n-\right.$ 1)) in the statistical weights of (17) by a mean field $\sigma_{\omega}$. A self-consistent description requires to formulate simultaneously the equations for partition function and $\sigma_{\omega}$ :

$$
\begin{aligned}
\mathcal{Z}_{\omega}^{\mathrm{MFA}} & \equiv \mathrm{e}^{-x} \sum_{n=0}^{\infty} z^{n} \exp \left(x \sigma_{\omega}\right), \\
\sigma_{\omega} & \equiv \frac{\mathrm{e}^{-x}}{\mathcal{Z}_{\omega}^{\mathrm{MFA}}} \sum_{n=0}^{\infty} \frac{1-\mu_{\omega}}{1+\mu_{\omega}(n-1)} z^{n} \exp \left(x \sigma_{\omega}\right) .
\end{aligned}
$$

One obtains

$$
\mathcal{Z}_{\omega}^{\mathrm{MFA}}=\frac{\mathrm{e}^{x\left(\sigma_{\omega}-1\right)}}{1-z}, \quad \sigma_{\omega}=\Lambda\left(z, \mu_{\omega}\right)
$$

where the function

$$
\Lambda(z, \mu)=\frac{(1-\mu)(1-z)}{\mu} \Phi\left(z, 1, \mu^{-1}-1\right)
$$

is expressed analytically through the Lerch transcendent

$$
\Phi(z, s, a)=\sum_{n=0}^{\infty} \frac{z^{n}}{(a+n)^{s}}
$$


and visualized by the dashed curves in Fig. 1 . Note that $\sigma_{\omega}$ is equal to $\sigma\left(z, 0, \mu_{\omega}\right)$, and the model equations in MFA are fairly simple to handle.

To relate $\mathcal{Z}_{\omega}^{\mathrm{MFA}}$ with $\mathcal{Z}_{\omega}$ let us expand $\mathcal{Z}_{\omega}=\mathrm{e}^{-x} \sum_{n} z^{n} \exp \left(x \sigma_{\omega}+x s_{\omega, n}\right)$ into the series over fluctuations $s_{\omega, n}=\left(1-\mu_{\omega}\right) /\left(1+\mu_{\omega}(n-1)\right)-\sigma_{\omega}$. A simple algebra leads to the formula:

$$
\mathcal{Z}_{\omega}=\mathcal{Z}_{\omega}^{\mathrm{MFA}}+\mathrm{e}^{x\left(\sigma_{\omega}-1\right)} \sum_{k=1}^{\infty}\left(-x \sigma_{\omega}\right)^{k} \sum_{m=0}^{k} \frac{\left(-\mu_{\omega} \sigma_{\omega}\right)^{-m}\left(1-\mu_{\omega}\right)^{m}}{m !(k-m) !} \Phi\left(z, m, \mu_{\omega}^{-1}-1\right),
$$

where the binomial expansion and the definition of $\Phi(z, s, a)$ have been used. Note that the term indexed by $k=1$ vanishes due to (21).

Single-Mode Thermodynamic Functions. Computing the main thermodynamic functions, we limit ourselves by the first non-vanishing terms of expansion in small parameter $x=\beta \kappa$, depending explicitly on $T$. That yields

$$
\begin{aligned}
\mathcal{N}_{\omega} & =\left(z \partial_{z} \ln \mathcal{Z}_{\omega}^{\mathrm{MFA}}\right)_{\beta, \sigma_{\omega}}+O(x) \\
& =\frac{z}{1-z}+O(x), \\
U_{\omega} & =-\left(\partial_{\beta} \ln \mathcal{Z}_{\omega}^{\mathrm{MFA}}\right)_{z, \sigma_{\omega}}+O(x) \\
& =\kappa\left[1-\Lambda\left(z, \mu_{\omega}\right)\right]+O(x), \\
S_{\omega} & =\left(\ln \mathcal{Z}_{\omega}^{\mathrm{MFA}}-x \partial_{x} \ln \mathcal{Z}_{\omega}^{\mathrm{MFA}}\right)_{z, \sigma_{\omega}}+O(x) \\
& =-\ln (1-z)+O(x) . \\
C_{\omega} & =\left(x^{2} \partial_{x}^{2} \ln \mathcal{Z}_{\omega}^{(2)}\right)_{z, \sigma_{\omega}}+O\left(x^{3}\right),
\end{aligned}
$$

where $\mathcal{N}_{\omega}=\left(z \partial_{z} \ln \mathcal{Z}_{\omega}\right)_{\beta}, U_{\omega}=-\left(\partial_{\beta} \ln \mathcal{Z}_{\omega}\right)_{z}$ and $S_{\omega}=\left(\ln \mathcal{Z}_{\omega}-x \partial_{x} \ln \mathcal{Z}_{\omega}\right)_{z}$ are the total mean number of photons, the total internal energy and the entropy, respectively. These functions are immediately evaluated in MFA. Numerical tests confirm a good agreement of $\mathcal{N}_{\omega}(z)$ from (25) with exact dependencies, obtained at $x \ll 1$.

The specific heat $C_{\omega}$ requires the use of a partition function in the next to leading-order approximation:

$$
\begin{aligned}
\mathcal{Z}_{\omega}^{(2)}\left(z, x, \mu_{\omega}, \sigma_{\omega}\right)= & \mathcal{Z}_{\omega}^{\mathrm{MFA}}\left(z, x, \sigma_{\omega}\right)\left[1+F\left(z, x, \mu_{\omega}, \sigma_{\omega}\right)\right] \\
F\left(z, x, \mu_{\omega}, \sigma_{\omega}\right) \equiv & \frac{x^{2}}{2}\left[\frac{\left(1-\mu_{\omega}\right)^{2}(1-z)}{\mu_{\omega}^{2}} \Phi\left(z, 2, \mu_{\omega}^{-1}-1\right)\right. \\
& \left.-2 \sigma_{\omega} \Lambda\left(z, \mu_{\omega}\right)+\left(\sigma_{\omega}\right)^{2}\right]+x\left[\Lambda\left(z, \mu_{\omega}\right)-\sigma_{\omega}\right],
\end{aligned}
$$

where the function $F$ is assumed to be a small correction.

From $\ln \mathcal{Z}_{\omega}^{(2)}=\ln \mathcal{Z}_{\omega}^{\mathrm{MFA}}+F$ (linear approximation in $F$ ), we also find

$$
C_{\omega}=x^{2} v\left(z, \mu_{\omega}\right)+O\left(x^{3}\right),
$$

where the function $v(z, \mu)=\partial_{x}^{2} F(z, x, \mu, \Lambda(z, \mu))$ is

$$
v(z, \mu)=\frac{(1-\mu)^{2}(1-z)}{\mu^{2}} \Phi\left(z, 2, \mu^{-1}-1\right)-\Lambda^{2}(z, \mu) .
$$



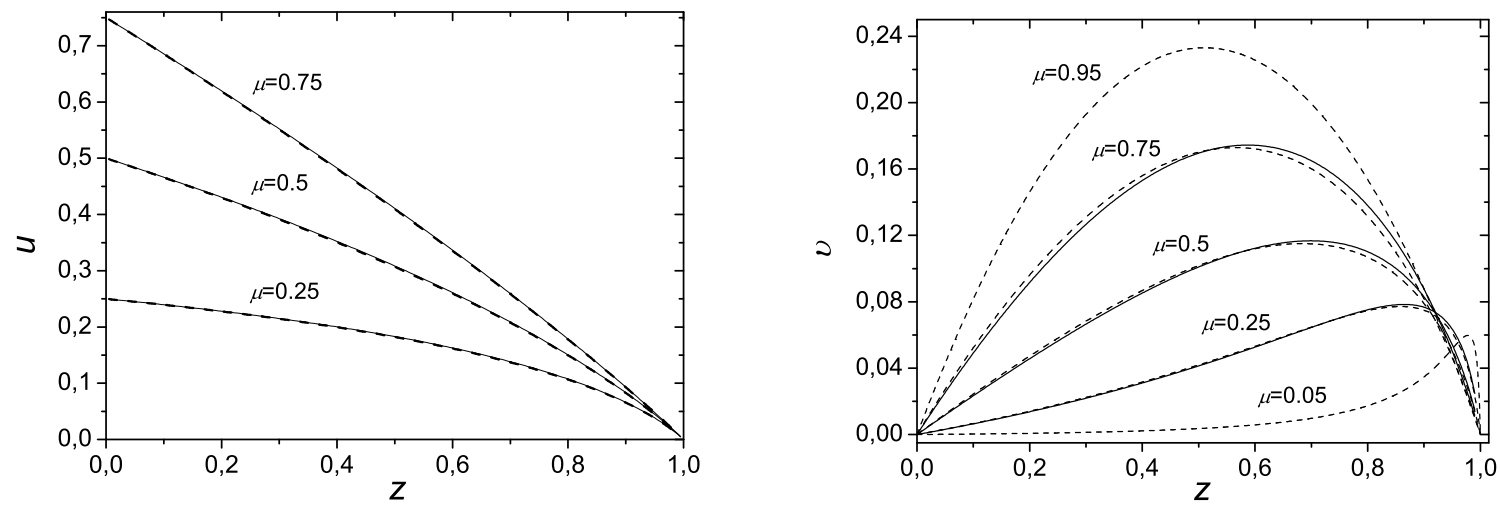

Figure 2: Dimensionless internal energy $u_{\omega}$ per photon (left panel) and variance $v$ (right panel) vs fugacity $z$ at some $\mu$. Solid curves represent the exact dependencies (34) and (32) at $\beta \kappa=0.1$. Dashed curves are analytically calculated in MFA.

One can see that it coincides with the variance

$$
\left\langle\left(\frac{1-\mu}{1+\mu(n-1)}\right)^{2}\right\rangle_{\mathrm{MFA}}-\left\langle\frac{1-\mu}{1+\mu(n-1)}\right\rangle_{\mathrm{MFA}}^{2} .
$$

Note that $C_{\omega} \equiv\left(-\beta^{2} \partial_{\beta} U_{\omega}\right)_{z, \sigma_{\omega}}=0$ results from substituting $U_{\omega}$ from (26) or taking $x=0$ in (31).

Analyzing, it is useful to compare the dimensionless internal energy per photon $u_{\omega}=$ $U_{\omega} /\left(\kappa \mathcal{N}_{\omega}\right)$, derived from (26) and (25), with exact function

$$
u\left(z, x, \mu_{\omega}\right)=\frac{\mu_{\omega} \sum_{n=0}^{\infty} n\left[1+\mu_{\omega}(n-1)\right]^{-1} w_{n}\left(z, x, \mu_{\omega}\right)}{\sum_{n=0}^{\infty} n w_{n}\left(z, x, \mu_{\omega}\right)},
$$

defined by the reduced statistical weights:

$$
w_{n}(z, x, \mu)=z^{n} \exp \left(x \frac{1-\mu}{1+\mu(n-1)}\right) .
$$

Actually, $u_{\omega}$ (in MFA) and $u\left(z, x, \mu_{\omega}\right)$ at $x=0.1$ are seen to coincide in Fig. 2 (left), that confirms validity of our approach in the regime of high- $T$. Since the total internal energy $U_{\omega}$ increases from 0 to $\kappa$ at $z \rightarrow 1$, with tending $\mathcal{N}_{\omega} \rightarrow \infty$, this explains the vanishing $u_{\omega}$ (and $\left.u\left(z, x, \mu_{\omega}\right)\right)$ at $z \rightarrow 1$. We also see that the internal energy per photon $\kappa u_{\omega}$ tends to $\hbar \omega$ at $z \rightarrow 0$.

Now consider the specific heat $C_{\omega}$ of one-mode subsystem. The behavior of $v(z, \mu)$ is seen in Fig. 2 (right), where its peaks at points $\left(z_{p}, \mu_{p}\right)$ imply large fluctuations which can be related to transient processes and are suppressed at $x \ll 1$. Therefore, we expect a presence of two phases in this model.

Note that from (32) it follows: $\lim _{\mu \rightarrow 1} v(z, \mu)=z(1-z)$. This at $\mu \rightarrow 1$ yields reflection symmetry with respect to the point $z=0.5$. 
Radiation Characteristics. Assuming that the volume $V$ is large enough, we may replace discrete energy spectrum by a continuous one. Then, the number of quantum states within the interval $(\omega, \omega+\mathrm{d} \omega)$ is given by $\left(V \omega^{2} / \pi^{2} c^{3}\right) \mathrm{d} \omega$, accounting for the two polarization directions of photons [23].

Due to the additivity property of the energies of different modes, the energy of radiation, accumulated within the frequency interval $(\omega, \omega+\mathrm{d} \omega)$ is $\mathrm{d} \mathcal{E}=U_{\omega}\left(V \omega^{2} / \pi^{2} c^{3}\right) \mathrm{d} \omega$. Thus, the spectral density of radiation,

$$
\frac{1}{4 \pi V} \frac{\mathrm{d} \mathcal{E}}{\mathrm{d} \omega}=\frac{\kappa^{3}}{4 \pi^{3} c^{3} \hbar^{2}} e\left(z, x, \mu_{\omega}\right)
$$

is determined by the following function derived from $\mathcal{Z}_{\omega}$ :

$$
e(z, x, \mu)=\mu^{3} \frac{\sum_{n=0}^{\infty} n[1+\mu(n-1)]^{-1} w_{n}(z, x, \mu)}{\sum_{n=0}^{\infty} w_{n}(z, x, \mu)} .
$$

Since the entities $\kappa \mu_{\omega}=\hbar \omega$ and $x \mu_{\omega}=\beta \hbar \omega$ are independent of $\kappa$, we have

$$
\lim _{\kappa \rightarrow \infty} \kappa^{3} e\left(z, x, \mu_{\omega}\right)=\frac{(\hbar \omega)^{3}}{z^{-1} \exp (\beta \hbar \omega)-1},
$$

that leads to the Planck formula for the spectral density of radiation [23].

Replacing summation over $\omega$ in (16) by integration with the upper bound $\omega_{\max }=\kappa / \hbar$ as argued above, the total energy of radiation is found to be

$$
\mathcal{E}=\frac{V \kappa^{4}}{\pi^{2}(\hbar c)^{3}} \varepsilon(z, x) .
$$

Here

$$
\varepsilon(z, x)=\int_{0}^{1} e(z, x, \mu) \mathrm{d} \mu, \quad \varepsilon_{0}(z)=\lim _{x \rightarrow 0} \varepsilon(z, x),
$$

is the dimensionless emitted energy which is plotted in Fig. 3.

Analytically, $\varepsilon_{0}(z)=\int_{0}^{1}[1-\Lambda(z, \mu)] \mu^{2} \mathrm{~d} \mu$ and equals

$$
\begin{aligned}
\varepsilon_{0}(z)= & \frac{z}{4}+\frac{z^{2}}{12}-z(1-z)\left(\frac{\operatorname{Li}_{1}(z)}{6}-\frac{\operatorname{Li}_{2}(z)}{2}-\operatorname{Li}_{3}(z)\right) \\
& -z(1-z) \sum_{k=1}^{\infty} \frac{z^{k}}{k^{4}}(k+1) \ln (k+1),
\end{aligned}
$$

where $\operatorname{Li}_{s}(z)$ is the polylogarithm (or Bose) function: $\operatorname{Li}_{s}(z)=\sum_{k=1}^{\infty} z^{k} / k^{s}$.

As seen from Fig. 3, at a fixed value $\bar{z}$ of fugacity the emitted total energy, taken in units of $V \kappa^{4} / \pi^{2}(\hbar c)^{3}$, is limited by (tends at $T \rightarrow \infty$ to) the respective value $\varepsilon_{0}(\bar{z})$. The special values of monotonic function $\varepsilon_{0}(z)$ are $\varepsilon_{0}(0)=0$ and $\varepsilon_{0}(1)=1 / 3$, what results from the definition of $\Lambda(z, \mu)$. 


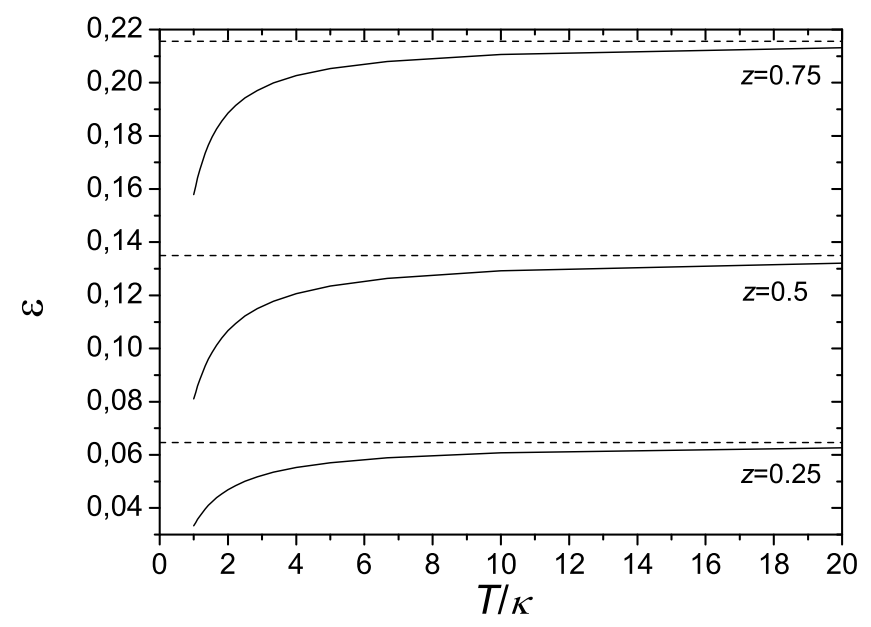

Figure 3: Temperature dependence of dimensionless emitted energy $\varepsilon$ from (40) at various $z$ (numerical results). Dashed lines correspond to the energy magnitudes at $T \rightarrow \infty$, determined by $\varepsilon_{0}(z)$ in (41).

Compare (39) with the Stefan-Boltzmann law [23]: $\mathcal{E}_{\mathrm{SB}}=\pi^{2} V T^{4} /\left(15 \hbar^{3} c^{3}\right)$. While $\mathcal{E}_{\mathrm{SB}} \rightarrow \infty$ at $T \rightarrow \infty$, the DSR based result predicts a finite value of the radiation energy at arbitrarily large temperature $T \gg \kappa$. As a similar feature, $\mathcal{E}_{\mathrm{SB}}$ and $\mathcal{E}$ represent the fourthorder law, defining the dependence of the total emitted energy on $T$ and $\kappa$ respectively. In the DSR based case, a kind of truncation of temperature $T$ due to presence of $\kappa$ is observed.

The total number of photons in black-body radiation, the total energy and the total entropy in MFA are calculated analytically on the base of (25)-(27):

$$
\begin{aligned}
\mathcal{N}^{\mathrm{MFA}} & =\frac{V \kappa^{3}}{3 \pi^{2}(\hbar c)^{3}} \frac{z}{1-z}, \\
\mathcal{E}^{\mathrm{MFA}} & =\frac{V \kappa^{4}}{\pi^{2}(\hbar c)^{3}} \varepsilon_{0}(z), \\
S^{\mathrm{MFA}} & =-\frac{V \kappa^{3}}{3 \pi^{2}(\hbar c)^{3}} \ln (1-z) .
\end{aligned}
$$

The equation of state is derived from the relation $\beta P V=\ln \mathcal{Z}^{\text {tot }}$. Using again the expressions in the MFA, one obtains

$$
P V=T S^{\mathrm{MFA}}-\mathcal{E}^{\mathrm{MFA}} .
$$

This formula differs from $P=\mathcal{E}_{\mathrm{SB}} /(3 V)$, which takes place for the usual ultrarelativistic particles. Unlike that, expanding the functions (42)-(45) into the series up to the first order in $z$ (when $0<z \ll 1$ for $-\ln z \sim \beta \kappa$ ), we obtain

$$
\tilde{\mathcal{N}}=\frac{V \kappa^{3}}{3 \pi^{2}(\hbar c)^{3}} z, \quad \tilde{\mathcal{E}}=\frac{V \kappa^{4}}{4 \pi^{2}(\hbar c)^{3}} z,
$$




$$
\tilde{S}=\frac{V \kappa^{3}}{3 \pi^{2}(\hbar c)^{3}} z, \quad \tilde{P}=\frac{\kappa^{4} z}{12 \pi^{2}(\hbar c)^{3}}\left(4 \frac{T}{\kappa}-3\right),
$$

so that the ultrarelativistic particle relations $\tilde{S}=4 \tilde{\mathcal{E}} /(3 T)$ and $\tilde{P}=\tilde{\mathcal{E}} /(3 V)$ do hold for $T=\kappa$. Also, $\tilde{S} / \tilde{\mathcal{N}}=$ const, in similarity with the usual photon gas [23].

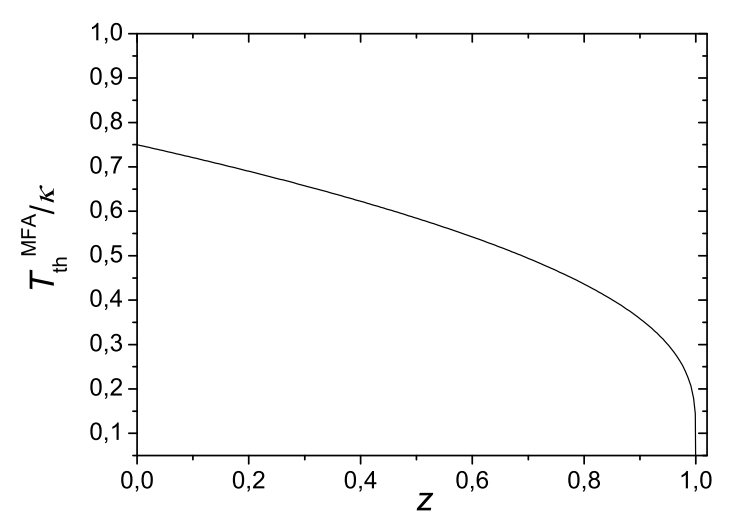

Figure 4: Temperature threshold of photon emission as function of $z$.

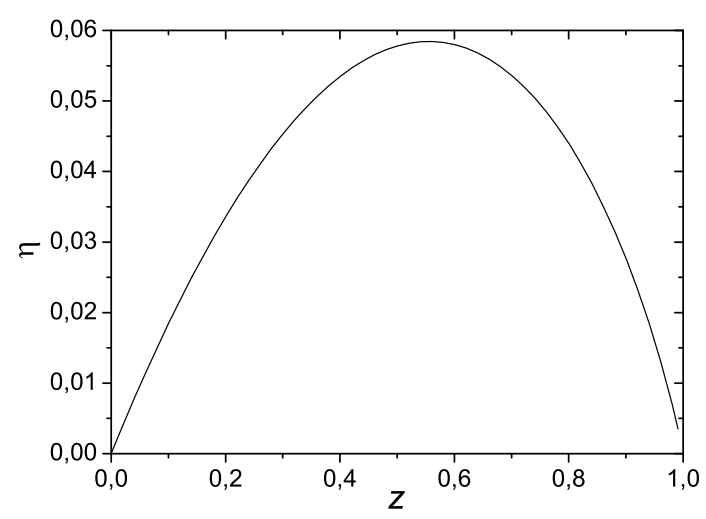

Figure 5: Function $\eta(z)$ by which the specific heat $C^{\mathrm{MFA}}$ depends on $z$.

However, the formulas (42)-(45) cannot reproduce the known expressions for blackbody radiation in the limit $\kappa \rightarrow \infty$ because they are obtained in the MFA, corresponding to the high temperature regime.

Emission ability in the model with effective attraction can be given by the condition $P \geq 0$, that is realized at the temperatures $T$ larger than a threshold $T_{\mathrm{th}}$. We find the temperature threshold $T_{\text {th }}$, when the system starts to radiate, by requiring $P=0$. Formula (45) allows us to evaluate $T_{\mathrm{th}}^{\mathrm{MFA}}$ as a function of fugacity $z$. Analytically, that results in

$$
\frac{T_{\mathrm{th}}^{\mathrm{MFA}}(z)}{\kappa}=-3 \frac{\varepsilon_{0}(z)}{\ln (1-z)}, \quad \lim _{z \rightarrow 0} \frac{T_{\mathrm{th}}^{\mathrm{MFA}}(z)}{\kappa}=\frac{3}{4},
$$

what is depicted in Fig. 4.

Integrating, the total specific heat in MFA is obtained from (31) as

$$
C^{\mathrm{MFA}}=\frac{V}{T^{2}} \frac{\kappa^{5}}{\pi^{2}(\hbar c)^{3}} \eta(z)
$$

where we define $\eta(z)=(1-z) \eta_{1}(z)-(1-z)^{2} \eta_{2}(z)$, and

$$
\eta_{\alpha}(z)=\int_{0}^{1}\left(\Phi\left(z, 3-\alpha, \mu^{-1}-1\right)\right)^{\alpha}(1-\mu)^{2} \mathrm{~d} \mu, \quad \alpha=1,2 .
$$

Functions $\eta_{1,2}$ can be analytically calculated and are written out in explicit form in Appendix. The result of calculations is presented in Fig. 5.

Analyzing, we notice that the specific heat of ordinary black-body radiation $C \sim V T^{3}$, while $C^{\mathrm{MFA}} \sim V \kappa^{5} T^{-2}$. However, such rather unusual behavior of $C^{\mathrm{MFA}}$ is inherent to the systems with a finite energy band [23] (similarly to magnetic systems), what has assumed at the beginning. 


\section{Conclusion}

In this paper, an original $\kappa$-addition rule inspired by the DSR has been proposed, exhibiting the crucial role played by the Planck energy scale $\kappa$ in all our treatment. That rule has naturally led us to the nonlinear Hamiltonian of self-interacting one-mode systems of photons. The adopted Hamiltonian, possessing essentially nonlinear (rational) dependence on the excitation number operator and combined with the assumption of independence of different modes, was taken as a starting point for the evaluation, within the framework of DSR, of main thermodynamic quantities of black-body radiation. Clearly, the presence of the scale $\kappa$ manifested its importance in our main results on the thermodynamic characteristics and their physical implications.

First of all, the energy of one-mode subsystem has the property that it lies entirely in the band of finite width, and the upper bound is determined by $\kappa$. This property influences all the other thermodynamic functions.

Next, as follows from Eq. (48) and is clearly shown in Fig. 4, within our approach a kind of threshold temperature $T_{\mathrm{th}}$ (depending on $z$ ) appears: it implies that just above these values $T_{\mathrm{th}}(z)$ the radiation is present. The disclosed property of the DSR-based black-body radiation may have important consequences and unexpected manifestations.

Also it is worth to emphasize the peculiar behavior of the one-mode specific heat and the total one (shown respectively in Fig. 2 and Fig. 5), as well as the unusual dependence on the temperature that was pointed out in the paragraph above Eq. (42).

An interesting equation of state is obtained which essentially differs from what is familiar in the standard physics of black-body radiation. We hope to explore its implications in a separate work.

It is worth to comment on some works on black-body physics based on deformed thermodynamics [24, 25, 26, 27, 28, 29] and compare their conclusions with the well-known handbook results [23] and with those presented above. In the mentioned papers, main novelty that appears due to deformation, consisted in some modification of pre-factors in the inferred versions of the Stefan-Boltzmann law. Besides, the Stefan's constant begins to depend on a parameter of deformation. The Wien displacement law is still preserved for the deformed Bose gas, though with certain inclusion of deformation parameter. In [28], the Planck formula for the deformed Bose gas is really different from the ordinary one: there appear some new terms in Planck's formula, which correspond to the "interactions" among photons. Similar to the case of ideal Bose gas, the total energy of the deformed Bose gas is proportional to the fourth power $T^{4}$. The peculiar feature is that the Stefan-Boltzmann constant turns out to be effectively reduced by the deformation.

In general, most of the results presented in our paper differ from those just mentioned in a principal way, namely what concerns the energy lying within a finite band, the peculiar behavior of specific heat, and the existence of threshold temperature for radiation switching. We hope to develop more specified applications of the obtained results for description of realistic objects in astrophysics and for effective modeling in modern cosmology.

Acknowledgement. This work was partly supported by the National Research Foundation of Korea Grant funded by the Korean Government (NRF-2015R1D1A1A01057792) 
and by Development Fund Foundation, Gyeongsang National University, 2018. Also, the work was partly supported by The National Academy of Sciences of Ukraine (project No. 0117U000237).

\section{Appendix: Computational Results}

Here we write down functions, defined by (150), as

$$
\begin{aligned}
\eta_{1}(z)= & \frac{1}{3}+\frac{z}{30}+\sum_{n=2}^{\infty}\left(\frac{n^{2}+10 n+1}{3(n-1)^{4}}-2 \frac{n(n+1)}{(n-1)^{5}} \ln n\right) z^{n} \\
\eta_{2}(z)= & \frac{1}{3}+\frac{z}{6}+\left(4 \ln 2-\frac{79}{30}\right) z^{2}+\sum_{n=3}^{\infty}\left(\frac{2 n}{(n-1)^{4}} \ln n\right. \\
& +2 \frac{(n-1)^{2}}{(n-2)^{5}} \ln (n-1)+\frac{1}{3(n-1)}-\frac{n+1}{(n-1)^{3}} \\
& \left.+\frac{n}{6(n-2)^{2}}-\frac{n(n-1)}{(n-2)^{4}}+A_{n}\right) z^{n} .
\end{aligned}
$$

Coefficient $A_{3}=0$ and $A_{n \geq 4}=\sum_{k=1}^{n-3} I(n-2-k, k)$, where

$$
\begin{aligned}
I(n, k)= & \int_{0}^{1} \frac{\mu^{2}(1-\mu)^{2}}{(1+\mu n)(1+\mu k)} \mathrm{d} \mu \\
= & \frac{(n+1)^{2}}{n^{4}(n-k)} \ln (n+1)-\frac{(k+1)^{2}}{k^{4}(n-k)} \ln (k+1) \\
& +\frac{1}{6 n^{3} k^{3}}\left[2 k^{2} n^{2}+9 k n(n+k)+6\left(n^{2}+k n+k^{2}\right)\right], n \neq k ; \\
I(n, n)= & \frac{n^{2}+12 n+12}{3 n^{4}}-2 \frac{(n+1)(n+2)}{n^{5}} \ln (n+1) .
\end{aligned}
$$

One finds that $I(n, n)=\lim _{k \rightarrow n} I(n, k)$, and $I(n-1, n-1)$ is already used as the series coefficient in (51).

\section{References}

[1] G. Amelino-Camelia, Int. J. Mod. Phys. D11 (2002) 35.

[2] G. Amelino-Camelia, Phys. Lett. B510 (2001) 255.

[3] J. Lukierski, A. Nowicki, H. Ruegg and V.N. Tolstoy, Phys. Lett. B264 (1991) 331.

[4] J. Lukierski, A. Nowicki and H. Ruegg, Phys. Lett. B293 (1992) 344.

[5] J. Magueijo and L. Smolin, Phys. Rev. Lett. 88 (2002) 190403. 
[6] S. Judes and M. Visser, Phys. Rev. D68 (2003) 045001.

[7] S. Meljanac, M. Milekovic and S.Pallua, Phys. Lett. B328 (1994) 55.

[8] A. Jannussis, J. Phys. A: Math. Gen. 26 (1993) L233.

[9] M. Arik and D.D. Coon, J. Math. Phys. 17 (1976) 524.

[10] L.C. Biedenharn, J. Phys. A: Math. Gen. 22 (1989) L873; A.J. Macfarlane, J. Phys. A: Math. Gen. 22 (1989) 4581.

[11] A. Chakrabarti and R. Jagannathan, J. Phys. A: Math. Gen. 24 (1991) L711; M. Arik et al., Z. Phys. C55 (1992) 89.

[12] W.S. Chung, K.S. Chung, S.T. Nam and C.I.Um, Phys. Lett. A183 (1993) 363.

[13] S.S. Mizrahi, J.P. Camargo Lima and V.V. Dodonov, J. Phys. A: Math. Theor. 37 (2004) 3707.

[14] I.M. Burban, Phys. Lett. A366 (2007) 308.

[15] A.M. Gavrilik, I.I. Kachurik and A.P. Rebesh, J. Phys. A: Math. Theor. 43 (2010) 245204.

[16] A.M. Gavrilik and A.P. Rebesh, Eur. Phys. J. A47 (2011) 55.

[17] A.M. Gavrilik and Yu.A. Mishchenko, Phys. Lett. A376 (2012) 2484.

[18] A.P. Rebesh, A.M. Gavrilik, I. Kachurik, Ukr. J. Phys. 58 (2013) 1182.

[19] A.M. Gavrilik, I.I. Kachurik, M.V. Khelashvili and A.V. Nazarenko, Physica A: Stat. Mech. and its Applics. 506 (2018) 835.

[20] G. Mandanici, Mod. Phys. Lett. A24 (2009) 739.

[21] S. Hossenfelder, SIGMA 10 (2014) 074.

[22] I.O. Vakarchuk, G.I. Panochko, Condens. Matter Phys. 3 (2015) 33002.

[23] R.B. Pathria, P.D. Beale, Statistical mechanics, Elsevier, Singapore, 2011.

[24] M.A. Martin-Delgado, J. Phys. A: Math. Gen. 24 (1991) L1285.

[25] P. Angelopoulou et al., J. Phys. A: Math. Gen. 27 (1994) L605.

[26] R. Gupta, C. Bach, H. Rosu, J. Phys. A: Math. Gen. 27 (1994) 1427.

[27] C. Tsallis, F.C. Sa Barreto and E.D. Loh, Phys. Rev. E52 (1995) 1447.

[28] Z. Chang, S.-X. Chen, J. Phys. A: Math. Gen. 35 (2002) 9731.

[29] X.-Y. Zhang, L.-J. Shao, B.-Q. Ma, Astroparticle Phys. 34 (2011) 840. 